\title{
ADSORÇÃO DO CORANTE REATIVO LARANJA 16 DE SOLUÇÕES AQUOSAS POR ZEÓLITA SINTÉTICA
}

\author{
Terezinha Elizabeth Mendes de Carvalho, Denise Alves Fungaro* e Juliana de Carvalho Izidoro \\ Centro de Química e Meio Ambiente, Instituto de Pesquisas Energéticas e Nucleares, Av. Prof. Lineu Prestes, 2242, 05508-000 \\ São Paulo - SP, Brasil
}

Recebido em 20/3/09; aceito em 22/7/09; publicado na web em 8/1/10

\begin{abstract}
ADSORPTION OF REACTIVE ORANGE 16 FROM AQUEOUS SOLUTIONS BY SYNTHESIZED ZEOLITE. Coal fly ash, a waste generated in a coal-fired electric power plant, was used to synthesize zeolite by hydrothermal treatment with $\mathrm{NaOH}$ solution. This zeolite was used as adsorbent to investigate the adsorption kinetics and isotherm parameters of the reactive orange 16 (RO16) dye from aqueous solutions at different concentrations (1.3-15.4 $\left.\mathrm{mg} \mathrm{L}^{-1}\right)$. Three kinetic models, the pseudo-first-order, second-order, and intraparticle diffusion were used to predict the adsorption rate constants. The kinetics of adsorption of the RO16 dye followed pseudo-second-order kinetics. The adsorption isotherm data were closely fitted to the Langmuir equation. Keywords: coal fly ash; zeolite; reactive dye adsorption.
\end{abstract}

Keywords: coal fly ash; zeolite; reactive dye adsorption.

\section{INTRODUÇÃO}

A matriz de energia elétrica no Brasil resulta de várias fontes, sendo uma delas proveniente de carvão mineral, que gera $1,31 \%$ da energia elétrica utilizada no país. ${ }^{1} \mathrm{Na}$ geração de eletricidade, sete usinas termelétricas dos Estados do sul do Brasil produzem aproximadamente 3 milhões de toneladas de cinzas a cada ano, as quais são compostas de 65 a $85 \%$ de cinza leve e 15 a $35 \%$ de cinza pesada. $^{2}$

O principal esforço para minimizar os impactos ambientais decorrentes da disposição destes resíduos no meio ambiente deve ser na busca de ampliar potencialidades para a sua utilização. ${ }^{3}$

No Brasil, a maior proporção de cinza leve é usada na produção de cimento pozolânico e em pavimentação, enquanto que a cinza pesada não tem, ainda, qualquer aplicação comercial. ${ }^{2}$ Há vários estudos para utilizar as cinzas em outras áreas como, por exemplo, na extração de metais de interesse industrial, aplicações cerâmicas, produção de geopolímeros e na síntese de várias zeólitas. ${ }^{4}$

A transformação das cinzas de carvão em adsorvente de baixo custo, capaz de remover corantes e íons metálicos tóxicos de efluentes aquosos, tem sido uma alternativa para reduzir os impactos ambientais decorrentes da sua disposição no meio ambiente..$^{5-10}$

O carvão ativado vem sendo largamente utilizado para remoção de corantes de efluentes devido à sua grande área superficial e grande capacidade de adsorção de compostos orgânicos, no entanto o alto custo tem limitado o seu uso. ${ }^{11,12}$

Daí o interesse pelo desenvolvimento de outros produtos com características adsorventes que possam utilizar o resíduo de usinas termelétricas a carvão como matéria-prima para o tratamento de efluente de indústrias que utilizem corantes.

A cinza leve é derivada de minerais contidos no carvão. No processo de combustão de carvão, esses minerais são parcialmente fundidos e formam partículas de cinzas leves nas quais as fases cristalinas, como quartzo $\left(\mathrm{SiO}_{2}\right)$ e mulita $\left(3 \mathrm{Al}_{2} \mathrm{O}_{3} \cdot 2 \mathrm{SiO}_{2}\right)$, permanecem no núcleo enquanto o aluminossilicato em fase vítrea cobre a superfície. ${ }^{4}$

O estudo da formação e da composição química e mineralógica de zeólitas naturais conduziu à síntese de zeólitas a partir de cinzas de carvão, porque os materiais precursores de zeólitas naturais e

\footnotetext{
*e-mail: dfungaro@ipen.br
}

cinzas de carvão são muito similares. ${ }^{13}$ Devido a essas similaridades, a síntese de zeólitas utilizando cinzas de carvão como matéria-prima vem sendo estudada por diversos pesquisadores..$^{14-16}$

As zeólitas são aluminossilicatos perfeitamente cristalinos de fórmula geral $\mathrm{M}_{2 / n} \mathrm{O} \cdot \mathrm{Al}_{2} \mathrm{O}_{3} . z \mathrm{SiO} 2$, onde $n$ é a valência do cátion $\mathrm{M}$ e $z$ pode variar entre 2 e infinito. Estruturalmente, as zeólitas são polímeros cristalinos baseados num arranjo tridimensional de tetraedros $\mathrm{TO}_{4}\left(\mathrm{SiO}_{4}\right.$ ou $\left.\mathrm{AlO}_{4}^{-}\right)$ligados pelos seus átomos de oxigênio para formar subunidades e, finalmente, enormes redes constituídas por blocos idênticos. ${ }^{17}$

Querol e colaboradores ${ }^{14}$ usaram $\mathrm{KOH}$ e $\mathrm{NaOH}$ como ativadores para sintetizar zeólita tanto em sistema aberto quanto em fechado. Realizaram experimentos com cinzas leves de variadas composições, coletadas em diferentes termelétricas. Os resultados indicaram melhor eficiência de conversão com as soluções de $\mathrm{NaOH}$ do que com as de $\mathrm{KOH}$, mesmo em concentrações maiores de $\mathrm{KOH}$. Indicaram também que, mesmo em concentração de $\mathrm{KOH} 1,0 \mathrm{~mol} \mathrm{~L}^{-1}$ a $200{ }^{\circ} \mathrm{C}$, o quartzo e a mulita permanecem na cinza leve.

A fusão alcalina é um método convencional de análise química para decompor materiais que contêm silício e/ou alumínio. O $\mathrm{NaOH}$ presente na mistura durante a reação atua como um ativador durante a fusão para formar sais solúveis de silicato e aluminatos, os quais posteriormente integram a formação da zeólita durante o processo hidrotérmico. ${ }^{18}$

A fase vítrea tem uma função importante na formação da zeólita, devido a sua grande solubilidade em solução alcalina. ${ }^{4}$ Nesta fase podem ser encontradas pequenas quantidades de metais tóxicos, mas que são separados no processo de síntese do material zeolítico., ${ }^{4,9}$

Levandowski e Kalkreuth ${ }^{2}$ analisaram cinzas leves da usina termelétrica de Figueira - PR e encontraram teores de $\mathrm{SiO}_{2}$ e $\mathrm{Al}_{2} \mathrm{O}_{3}$ entre 75 e $78 \%$ e de $\mathrm{Fe}_{2} \mathrm{O}_{3}$ entre 10 e 18\%, enquanto Fungaro e colaboradoras $^{8}$ analisaram zeólitas sintetizadas de cinzas de carvão de Figueira e encontraram conteúdos de $\mathrm{SiO}_{2}$ e $\mathrm{Al}_{2} \mathrm{O}_{3}$ de 65 a $70 \%$ e de $\mathrm{Fe}_{2} \mathrm{O}_{3}$ de 10 a $16 \%$. Embora os compostos predominantes na cinza e na zeólita sejam os mesmos, o que os diferenciam são suas estruturas cristalinas. ${ }^{18}$

A presença de corantes nas correntes aquosas reduz a atividade fotossintética, afetando o equilíbrio natural da biota. Efluentes que contêm corantes representam um sério problema ambiental, devido 
à sua grande toxicidade e possível acumulação no meio ambiente. ${ }^{19}$

Os corantes reativos são os mais comuns porque apresentam vantagens como cores brilhantes, estabilidade na cor e facilidade de aplicação. ${ }^{20}$ Eles têm sido um dos mais utilizados no Brasil para a tintura de algodão, devido à sua reatividade com as fibras e sua estabilidade de cor. $^{21}$ Estes corantes são considerados alérgenos ${ }^{22}$ e, sob certas condições, alguns são considerados mutagênicos e carcinógenos. $^{23,24}$

Métodos de adsorção têm sido usados com sucesso para descolorir efluentes têxteis, mas sua aplicação vem sendo limitada devido ao alto custo dos adsorventes. ${ }^{25}$ Por isso, a utilização de zeólita sintetizada a partir do resíduo gerado em termelétricas a carvão mineral vem sendo avaliada como uma solução de baixo custo.

Por comparação, o custo da preparação de zeólitas sintetizadas com cinzas de carvão revelou ser muito próximo do custo das zeólitas comerciais 4A e 5A. ${ }^{3}$ Segundo Biniwale e colaboradores, ${ }^{26}$ o custo de produção de zeólita de cinza de carvão indiano é de 25 a $30 \%$ menor do que aquele da zeólita comercial análoga tipo A. A análise de custo foi baseada em um processo de produção de $7500 \mathrm{~kg} / \mathrm{dia}$ de zeólita e o retorno do investimento foi estimado em um período de 42 meses. Maiores reduções de custo foram previstas quando a solução de $\mathrm{NaOH}$ da síntese é reutilizada. Outro estudo de custo em grande escala foi feito por Ojha e colaboradores, ${ }^{18}$ que revelou que o custo da produção de zeólita de cinza de carvão foi estimado como 1/5 do custo da zeólita 13X análoga disponível comercialmente.

Nesse estudo, avaliou-se a eficiência de adsorção do corante Reativo Laranja 16 (RL16) em solução aquosa sobre zeólita sintetizada a partir de cinzas leves de carvão mineral.

\section{PARTE EXPERIMENTAL}

\section{Materiais}

Todos os reagentes químicos usados foram de grau analítico e as soluções foram preparadas com água desionizada obtida pelo sistema Millipore Milli-Q.

\section{Síntese da zeólita}

A zeólita sintética (ZM-2) foi preparada a partir da cinza leve de carvão (CL-2) retida no filtro manga. A cinza leve foi coletada na Usina Termelétrica Carbonífera de Cambuí Ltda., localizada na cidade de Figueira, estado do Paraná. Uma solução de $\mathrm{NaOH} 3,5$ mol L ${ }^{-1}$ foi adicionada à cinza leve de carvão na razão $1 / 8(\mathrm{~m} / \mathrm{v})$ e a mistura mantida em estufa a $100{ }^{\circ} \mathrm{C}$ por $24 \mathrm{~h}$. A suspensão foi filtrada e o resíduo lavado repetidamente com água desionizada até $\mathrm{pH} \sim 10$. O resíduo foi seco em estufa a $50{ }^{\circ} \mathrm{C}$ por $12 \mathrm{~h}$.

\section{Corante Reativo Laranja 16 (RL16)}

O RL16, cuja fórmula molecular é $\mathrm{C}_{20} \mathrm{H}_{17} \mathrm{~N}_{3} \mathrm{Na}_{2} \mathrm{O}_{11} \mathrm{~S}_{3}$ (adquirido da empresa Sigma-Aldrich com pureza de 50\%) tem o grupo azo como cromóforo (Tabela $1 \mathrm{~S}$ e Figura $1 \mathrm{~S}$, material suplementar). O comprimento de onda de absorção do corante a $493 \mathrm{~nm}$, o de maior intensidade de absorbância, foi escolhido para monitorar o processo de adsorção (espectrofotômetro UV/Vis Varian Cary 1E). O coeficiente de correlação da curva de calibração da solução aquosa em concentrações de 0 a $15,8 \mathrm{mg} \mathrm{L}^{-1}$ foi 0,999 .

\section{Caracterização da zeólita de cinzas de carvão}

A zeólita foi caracterizada por fluorescência de raios-X (Rigaku, RIX-3000), difração de raios-X (Rigaku, Multiflex), área superfi- cial específica BET (Quanta-Chrome Corp., Nova 1200) e massa específica (pictômetro Micromeritcs - Accupyc 1330). ${ }^{3}$ A técnica de infravermelho com transformada de Fourier (FTIR) foi usada para examinar os grupos funcionais principais da cinza e da zeólita sintetizada. Os espectros foram feitos no espectrofotômetro Nexus 670 FTIR da Thermo Nicolet.

\section{Estudos de adsorção}

A cinética de adsorção em sistema de batelada foi realizada com três soluções aquosas de RL16, de concentrações 5,0; 10,1 e 14,6 mg $\mathrm{L}^{-1}$; com pH 5 e temperatura ambiente de $25 \pm 2{ }^{\circ} \mathrm{C}$. Uma alíquota de $25 \mathrm{~mL}$ de cada solução foi adicionada a $0,25 \mathrm{~g}$ de adsorvente e a suspensão foi agitada a $120 \mathrm{rpm}$ (mesa agitadora Q225M) por intervalos de tempo entre 10 e $240 \mathrm{~min}$. Ao final de cada período de tempo desejado, $10 \mathrm{~mL}$ foram retirados e a solução de corante foi separada do adsorvente, por centrifugação (centrífuga Solumix), a $3000 \mathrm{rpm}$ por $3 \mathrm{~min}$. Uma porção do sobrenadante foi analisada por espectrofotometria UV/Vis em $\lambda=493 \mathrm{~nm}$, após ajuste para $\mathrm{pH} 5$ (pH da solução aquosa do corante) com $\mathrm{HNO}_{3} 0,05 \mathrm{M}$.

A eficiência de adsorção foi calculada usando-se a Equação 1:

$\mathrm{R}=\frac{100\left(\mathrm{C}_{0}-\mathrm{C}_{\mathrm{f}}\right)}{\mathrm{C}_{0}}$

onde R é a eficiência de adsorção (\%), $\mathrm{C}_{0}$ é a concentração inicial de corante RL16 (mg L $\left.{ }^{-1}\right)$ e $\mathrm{C}_{\mathrm{f}}$ é a concentração final de corante RL16 no tempo $\mathrm{t}\left(\mathrm{mg} \mathrm{L}^{-1}\right)$.

A quantidade do corante RL16 adsorvido na fase adsorvente foi quantificada conforme a Equação 2:

$\mathrm{q}_{\mathrm{e}}=\frac{V\left(C_{o}-C_{e}\right)}{M}$

onde $\mathrm{C}_{0}$ é a concentração inicial de corante RL $16\left(\mathrm{mg} \mathrm{L}^{-1}\right), \mathrm{C}_{\mathrm{e}}$ é a concentração de corante RL16 no tempo de equilíbrio $\left(\mathrm{mg} \mathrm{L}^{-1}\right)$, V é o volume da solução de corante RL16 (L) e M é a massa do adsorvente $(\mathrm{g})$.

As isotermas de adsorção foram determinadas, para a faixa de concentração de 1,3 a $15,4 \mathrm{mg} \mathrm{L}^{-1}$, adicionando-se $25 \mathrm{~mL}$ de solução de RL16 em 0,25 g de ZM-2 e submetendo a suspensão a agitação de $120 \mathrm{rpm}$ por $120 \mathrm{~min}$, que é o tempo necessário para alcançar o equilíbrio.

\section{RESULTADOS E DISCUSSÃO}

\section{Caracterização do material adsorvente}

A zeólita de cinzas de carvão constituía-se principalmente de alumina $(21,9 \%)$ e sílica $(44,0 \%)$. O óxido férrico $(12,8 \%)$ e o óxido de cálcio $(5,19 \%)$ estavam presentes em conteúdos baixos. Encontraram-se os óxidos de potássio, titânio, enxofre, magnésio e outros compostos em quantidades menores que 5,19\%. A área superficial BET e a massa específica apresentaram valores de $53,4 \mathrm{~m}^{2} / \mathrm{g}$ e 2,54 $\mathrm{g} / \mathrm{cm}^{3}$, respectivamente.

A Figura $2 \mathrm{~S}$ (material suplementar) mostra os difratogramas da zeólita ZM-2 e da cinza CL-2 que serviu como matéria-prima na síntese. As espécies cristalinas identificadas para a cinza são quartzo e mulita. O produto zeolítico formado foi identificado como hidroxissodalita (JCPDS 011-0401). Observou-se também a presença de quartzo e mulita provenientes das cinzas de carvão que não foram convertidas no tratamento hidrotérmico, porém o processo causou um decréscimo dos picos evidenciado principalmente em $26,8^{\circ}$. 
A micrografia MEV da zeólita está apresentada na Figura 3S (material suplementar). A superfície é rugosa, apresentando aglomerados de partículas pequenas porque os cristais de zeólita precipitam sobre as partículas das cinzas de carvão durante a síntese. As impurezas presentes na cinza de carvão causam uma nucleação rápida, prejudicando o crescimento dos cristais de zeólita. ${ }^{27}$

O espectro da análise por espectroscopia de infravermelho (IV) da ZM-2 é mostrado na Figura 4S (material suplementar), onde se encontra também o espectro da CL-2 para comparação.

O IV da CL-2 apresenta frequências características das vibrações relacionadas ao estiramento assimétrico das ligações $(\rightarrow \mathrm{OTO} \rightarrow \sim 1059$ $\left.\mathrm{cm}^{-1}\right)$, ao estiramento simétrico $\left(\leftarrow \mathrm{OTO} \rightarrow \sim 777 \mathrm{~cm}^{-1}\right)$ e à deformação angular ( $\mathrm{T}-\mathrm{O} \sim 455 \mathrm{~cm}^{-1}$ ), com $\mathrm{T}$ representando $\mathrm{Si}$ ou Al do material aluminossilicato amorfo e das fases cristalinas quartzo e mulita ${ }^{28} \mathrm{e}$ representando o oxigênio. O IV da ZM-2 apresenta uma banda forte em $\sim 993 \mathrm{~cm}^{-1}$, também atribuída ao estiramento assimétrico da ligação $\rightarrow \mathrm{OTO} \rightarrow$. A região central do infravermelho contém as vibrações das estruturas fundamentais dos grupamentos $\mathrm{Si}(\mathrm{Al}) \mathrm{O}_{4}$. Essas bandas são dependentes da estrutura cristalina. ${ }^{18}$ As informações dos espectros de infravermelho confirmam a mudança estrutural com o tratamento hidrotérmico da cinza leve.

\section{Estudos cinéticos}

A Figura 1 mostra o espectro de absorção, entre 400 e 600 nm, da solução aquosa do RL16 no tempo inicial $(\mathrm{t}=0$, remoção $=0 \%)$ e os espectros após ter ocorrido o processo de adsorção nos tempos pré-determinados. A análise dos espectros indica que a única variação que ocorreu no comportamento do RL16 foi o aumento da descoloração com o decorrer do tempo.

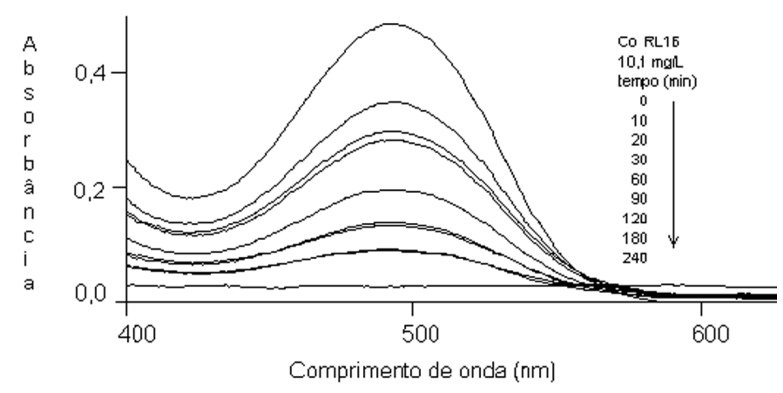

Figura 1. Espectro na região do visível para o RL16 antes e após adsorção sobre ZM-2 em diferentes tempos de contato

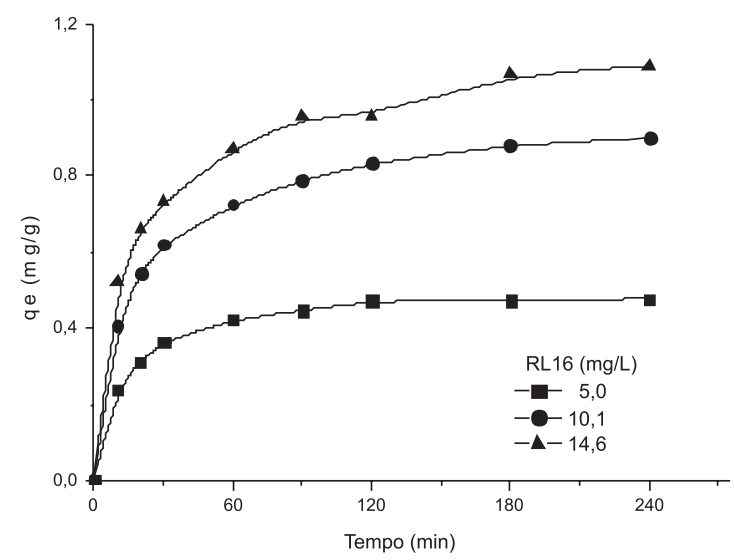

Figura 2. Efeito do tempo de agitação e da concentração sobre a capacidade de adsorção do RL16 sobre ZM-2
A Figura 2 indica que houve maior remoção do corante RL16 nos períodos iniciais de contato. A porcentagem de remoção de corante pelo adsorvente foi rápida no início, mas diminuiu gradualmente com o tempo até alcançar o equilíbrio aos 120 min, onde houve remoção de cerca de 94, 82 e $65 \%$ para as concentrações iniciais de 5,0; 10,1 e 14,6 $\mathrm{mg} \mathrm{L}^{-1}$ de RL16, respectivamente, no adsorvente ZM-2.

A velocidade de remoção foi maior no início devido à maior área superficial disponível de adsorvente. O tempo de contato entre o adsorbato (RL16) e o adsorvente (ZM-2) é muito significante no tratamento de efluentes aquosos por adsorção. A remoção rápida do adsorbato e o alcance de equilíbrio em um período curto de tempo indicam que o adsorvente é eficiente. ${ }^{29} \mathrm{~A}$ forma simples e contínua da curva até a saturação sugere uma cobertura por monocamada de corante RL16 na superfície da ZM-2. ${ }^{9}$

O comportamento transiente do processo de adsorção foi analisado utilizando-se os modelos cinéticos de pseudoprimeira ordem, pseudossegunda ordem e difusão intrapartícula.

A equação cinética da pseudoprimeira ordem é largamente utilizada para prever a cinética de adsorção de corantes. ${ }^{30} \mathrm{~A}$ velocidade de adsorção pode ser determinada por uma expressão de velocidade de pseudoprimeira ordem dada por Lagergren para a adsorção em sistema líquido-sólido baseada na capacidade de adsorção do sólido. ${ }^{31}$ Lagergren assumiu que a velocidade de remoção do adsorbato com o tempo é diretamente proporcional à diferença na concentração de saturação e ao número de sítios ativos de sólido. A equação cinética de Lagergren é a mais usada para a adsorção de um adsorbato de uma solução aquosa. ${ }^{32}$

A forma linear da equação da pseudoprimeira ordem é dada pela Equação 3:

$\log _{10}\left(q_{e}-q\right)=\log _{10} q_{e}-k_{1} t / 2,303$

onde $\mathrm{q}_{\mathrm{e}}$ e q são as quantidades de RL16 adsorvidas ( $\left.\mathrm{mg} \mathrm{g}^{-1}\right)$ no equilíbrio e no tempo $\mathrm{t}(\mathrm{min})$, respectivamente; $\mathrm{k}_{1}$ é a constante de velocidade de adsorção $\left(\min ^{-1}\right)$. A constante $\mathrm{k}_{1}$ pode ser calculada a partir da inclinação da reta do gráfico $\log \left(\mathrm{q}_{\mathrm{e}} \mathrm{-q}\right) v s \mathrm{t}$.

Os dados cinéticos foram também analisados usando as cinéticas de pseudossegunda ordem desenvolvidas por Ho e colaboradores, ${ }^{33}$ onde a velocidade da reação é dependente da quantidade do soluto adsorvido na superfície do adsorvente e da quantidade adsorvida no equilíbrio. O modelo linear de pseudossegunda ordem pode ser representado por:

$\frac{\mathrm{t}}{\mathrm{q}}=\frac{1}{\mathrm{k}_{2} \mathrm{q}_{\mathrm{e}}^{2}}+\frac{\mathrm{t}}{\mathrm{q}_{\mathrm{e}}}$

onde $\mathrm{q}_{\mathrm{e}}$ e $\mathrm{q}_{\mathrm{t}}$ são as quantidades de RL16 adsorvidas $\left(\mathrm{mg} \mathrm{g}^{-1}\right)$ no equilíbrio e no tempo $\mathrm{t}(\mathrm{min}), \mathrm{k}_{2}$ é a constante de velocidade de pseudossegunda ordem $\left(\mathrm{g} \mathrm{mg}^{-1} \mathrm{~min}^{-1}\right)$.

O mecanismo do processo de adsorção definitivo pode não ser obtido pelos modelos cinéticos descritos, então, o modelo da difusão intrapartícula pode ser empregado. De acordo com Weber e Morris, ${ }^{34}$ se a difusão intrapartícula é o fator determinante da velocidade, a remoção do adsorbato varia com a raiz quadrada do tempo. Assim, o coeficiente de difusão intrapartícula $\left(\mathrm{k}_{\mathrm{di}}\right)$ pode ser determinado pela Equação 5:

$\mathrm{q}_{\mathrm{t}}=\mathrm{k}_{\mathrm{di}} \mathrm{t}^{1 / 2}+\mathrm{C}$

onde $\mathrm{q}_{\mathrm{t}}$ é a quantidade de RL16 adsorvida $\left(\mathrm{mg} \mathrm{g}^{-1}\right)$, $\mathrm{t}(\mathrm{min})$ é o tempo de agitação e $\mathrm{C}\left(\mathrm{mg} \mathrm{g}^{-1}\right)$ é uma constante relacionada com a resistência à difusão.

De acordo com a Equação 5, os valores de $\mathrm{k}_{\mathrm{di}}\left(\mathrm{mg} / \mathrm{g} \min ^{0,5}\right)$ e $\mathrm{C}$ podem ser obtidos pela inclinação e intersecção da curva do gráfico $\mathrm{q}_{\mathrm{t}} v_{s} \mathrm{t}^{1 / 2}$, respectivamente. Os valores de $\mathrm{C}$ dão uma idéia da espessura 
da camada limite, isto é, quanto maior for o valor de $\mathrm{C}$ maior será o efeito da camada limite. ${ }^{35}$ Há estudos que mostraram que o gráfico pode apresentar uma multilinearidade, a qual caracteriza os diferentes estágios na adsorção: transferência de massa externa seguida por difusão intrapartícula no macro, meso e microporo. ${ }^{36}$

A Tabela 1 mostra os parâmetros cinéticos do processo de adsorção do RL16 pela ZM-2, os quais foram obtidos pelas regressões lineares das curvas de cada modelo (Figura 3 ).
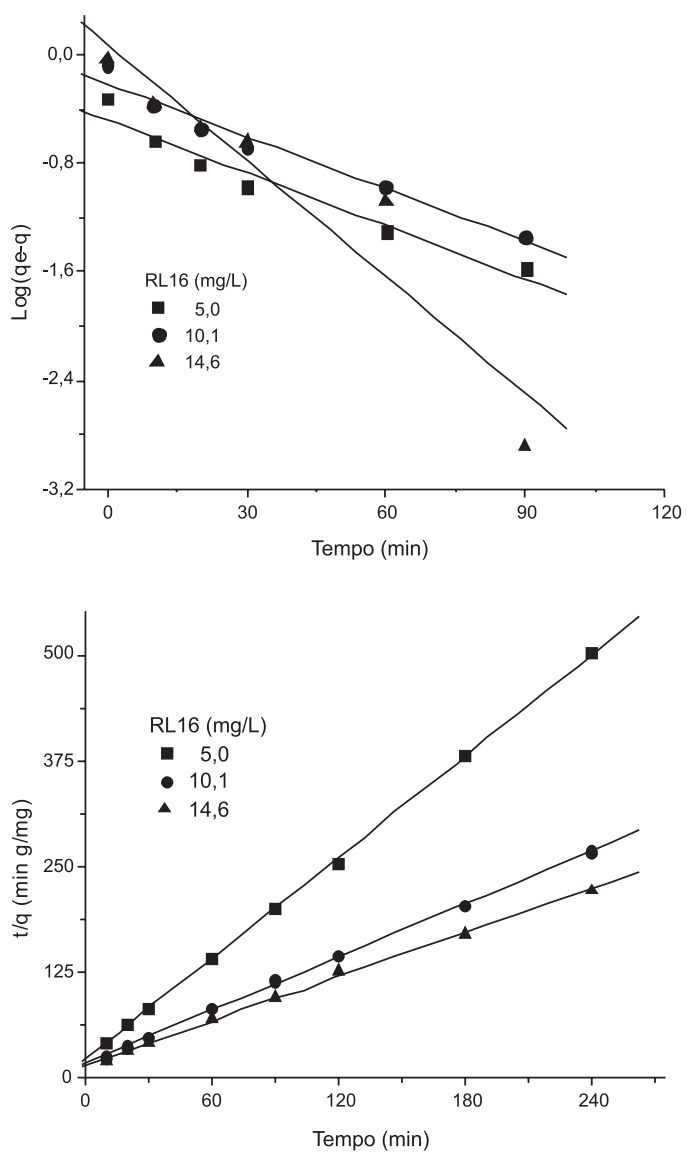

(b)

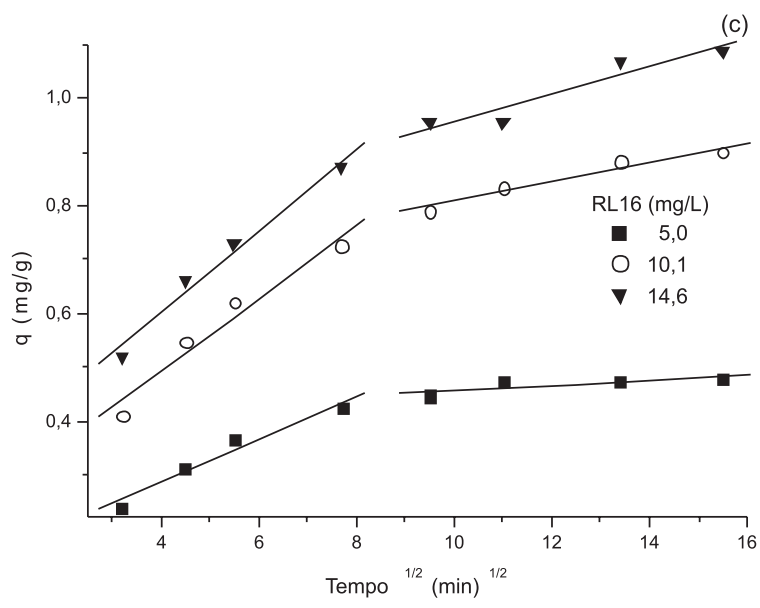

Figura 3. Modelos cinéticos da adsorção do RL16 sobre ZM-2: (a) pseudoprimeira ordem; (b) pseudossegunda ordem; (c) difusão intrapartícula

A avaliação quantitativa dos modelos foi realizada pela comparação dos coeficientes de determinação $\left(\mathrm{R}^{2}\right)$ e pelos $\mathrm{q}_{\mathrm{e}}$ calculados $v s$ os $\mathrm{q}_{\mathrm{e}}$ obtidos experimentalmente para os modelos de pseudoprimeira $\mathrm{e}$ pseudossegunda ordem. Os resultados mostraram que o processo de adsorção se ajustou melhor ao mecanismo de pseudossegunda ordem. Consequentemente, no controle da velocidade deve estar envolvido um mecanismo de adsorção ativado ou quimissorção. ${ }^{37}$

A Figura $3 c$ mostra que as linhas do gráfico $\mathrm{q}_{\mathrm{t}} v s \mathrm{t}^{1 / 2}$ não passam pela origem, indicando que o mecanismo de difusão intrapartícula não é a única etapa limitante do processo de adsorção e que outros mecanismos de interação devem estar atuando simultaneamente. Observa-se também que há duas linearidades, onde a primeira representa a adsorção instantânea ou adsorção na superfície externa e a segunda, ao estágio de adsorção gradual por difusão intrapartícula. ${ }^{38}$

Tabela 1. Parâmetros cinéticos para a remoção do RL16 pela ZM-2

\begin{tabular}{|c|c|c|c|c|c|}
\hline & \multicolumn{5}{|c|}{ Pseudo- $1^{\text {a }}$ ordem } \\
\hline $\begin{array}{l}\text { RL16 } \\
(\mathrm{mg} / \mathrm{L})\end{array}$ & $\begin{array}{l}\mathrm{k}_{1} \times 10^{-2} \\
\left(\min ^{-1}\right)\end{array}$ & $\begin{array}{c}\mathrm{qe}_{\text {calc. }} \times 10^{-1} \\
(\mathrm{mg} / \mathrm{g})\end{array}$ & $\begin{array}{c}\mathrm{qe}_{\text {exp. }} \times 10^{-1} \\
(\mathrm{mg} / \mathrm{g})\end{array}$ & \multicolumn{2}{|c|}{$\mathrm{R}_{1}^{2}$} \\
\hline 5,0 & 3,01 & 3,35 & 4,74 & \multicolumn{2}{|c|}{0,954} \\
\hline 10,1 & 2,97 & 6,19 & 8,34 & \multicolumn{2}{|c|}{0,970} \\
\hline \multirow[t]{3}{*}{14,6} & 6,60 & 12,2 & 9,57 & & \\
\hline & \multicolumn{5}{|c|}{ Pseudo-2 ${ }^{\mathrm{a}}$ ordem } \\
\hline & $\begin{array}{c}\mathrm{k}_{2} \times 10^{-2} \\
(\mathrm{~g} / \mathrm{mg} \min )\end{array}$ & $\begin{array}{c}\mathrm{h} \times 10^{-2} \\
(\mathrm{mg} / \mathrm{g} \min )\end{array}$ & $\begin{array}{c}\mathrm{qe}_{\text {calc. }} \times 10^{-1} \\
(\mathrm{mg} / \mathrm{g})\end{array}$ & $\begin{array}{c}\mathrm{qe}_{\text {exp. }} \times 10^{-1} \\
(\mathrm{mg} / \mathrm{g})\end{array}$ & $\mathrm{R}_{2}{ }^{2}$ \\
\hline 5,0 & 18,3 & 4,61 & 5,02 & 4,74 & 0,999 \\
\hline 10,1 & 6,46 & 5,90 & 9,55 & 8,34 & 0,999 \\
\hline \multirow[t]{3}{*}{14,6} & 5,13 & 6,81 & 11,5 & 9,57 & 0,998 \\
\hline & \multicolumn{5}{|c|}{ Difusão Intrapartícula } \\
\hline & $\begin{array}{l}\mathrm{C} \times 10^{-1} \\
(\mathrm{mg} / \mathrm{g})\end{array}$ & $\mathrm{R}_{\mathrm{di}, 1}^{2}$ & $\begin{array}{c}\mathrm{k}_{\mathrm{di}, 2} \times 10^{-3} \\
\left(\mathrm{mg} / \mathrm{g} \min ^{1 / 2}\right)\end{array}$ & & \\
\hline 5,0 & 1,27 & 0,980 & 4,39 & \multicolumn{2}{|c|}{0,804} \\
\hline 10,1 & 2,19 & 0,978 & 18,3 & \multicolumn{2}{|c|}{0,972} \\
\hline 14,6 & 2,97 & 0,991 & 25,5 & \multicolumn{2}{|c|}{0,952} \\
\hline
\end{tabular}

\section{Isoterma de adsorção}

Os dados de adsorção são comumente representados por uma isoterma de adsorção, a qual mostra relação de equilíbrio entre a quantidade do material adsorvido e a concentração na fase fluida em temperatura constante. ${ }^{39} \mathrm{~A}$ forma do gráfico da isoterma é determinada pelo mecanismo de adsorção e pode ser usada para sugerir o tipo de adsorção que ocorre entre o adsorvente e o adsorbato. ${ }^{40}$

A isoterma de adsorção para solução pode ser classificada em quatro principais classes, as quais são relacionadas de acordo com suas formas como, S, L, H e C e subgrupos 1, 2, 3, 4 ou max. A isoterma do tipo $\mathrm{S}$ sugere "adsorção cooperativa", a qual ocorre se a interação adsorbato-adsorbato é mais forte que a interação adsorbato-adsorvente; a do tipo L (Langmuir) reflete uma afinidade relativamente alta entre o adsorbato e o adsorvente e, normalmente, é indicativa de processo de quimissorção. A isoterma do tipo $\mathrm{H}$ indica forte interação entre o adsorbato e o adsorvente (isto é, quimissorção) e ocorre em casos extremos da isoterma do tipo L. A isoterma do tipo C (constante de partição) sugere uma afinidade relativa constante das moléculas do adsorbato com o adsorvente. ${ }^{41,42}$

No caso do processo de adsorção do RL16 com a ZM-2, a forma da isoterma (Figura 4) sugere um comportamento concordante com o tipo L2, no qual a afinidade de adsorção aumenta com o aumento da concentração do adsorbato até a saturação.

Os dados da isoterma foram analisados utilizando as isotermas de Langmuir e Freundlich. O modelo de adsorção de Langmuir baseiase na suposição que a adsorção máxima corresponde à monocamada 
saturada de moléculas de soluto na superfície do adsorvente, sem que haja qualquer interação lateral entre as moléculas adsorvidas. ${ }^{43}$ Este modelo foi desenvolvido presumindo que o adsorvente possui um número limitado de posições disponíveis na superfície e que as moléculas podem ser adsorvidas até que os sítios superficiais disponíveis estejam ocupados. Considera-se que as moléculas serão adsorvidas apenas nos sítios livres.

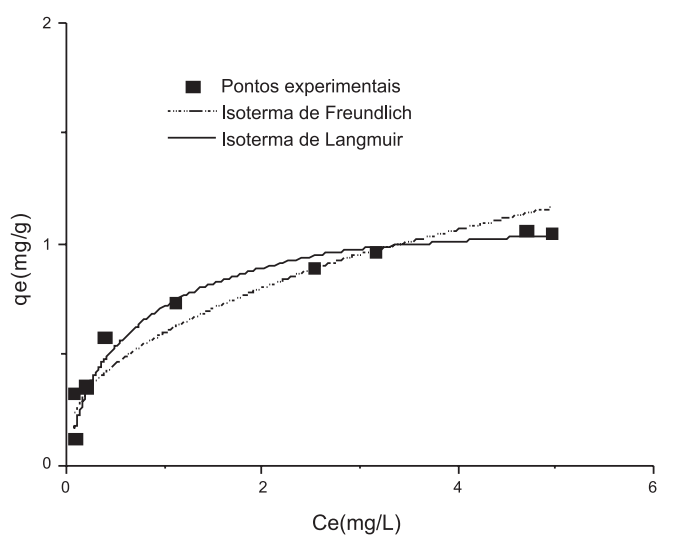

Figura 4. Isoterma de adsorção do RL16 sobre a $\mathrm{ZM}-2\left(T=25^{\circ} \mathrm{C} ; \mathrm{pH}=5\right)$

Teoricamente, alcança-se um valor de saturação além do qual não ocorre mais a adsorção. As moléculas são adsorvidas e aderem à superfície adsorvente em monocamada. ${ }^{29,44}$

A expressão linear de Langmuir é representada pela Equação 6:

$\frac{\mathrm{C}_{\mathrm{e}}}{\mathrm{q}_{\mathrm{e}}}=\frac{1}{\mathrm{Q}_{0} \mathrm{~b}}+\frac{\mathrm{C}_{\mathrm{e}}}{\mathrm{Q}_{0}}$

onde $\mathrm{C}_{\mathrm{e}}$ é a concentração de RL16 adsorvida no tempo de equilíbrio $\left(\mathrm{mg} \mathrm{L}^{-1}\right), \mathrm{q}_{\mathrm{e}}$ é a quantidade de RL16 no tempo de equilíbrio $\left(\mathrm{mg} \mathrm{g}^{-1}\right)$ $\mathrm{e}, \mathrm{Q}_{0}\left(\mathrm{mg} \mathrm{g}^{-1}\right)$ e b $\left(\mathrm{L} \mathrm{mg}^{-1}\right)$ são constantes relacionadas com a capacidade de adsorção máxima e a energia de adsorção, respectivamente.

A isoterma de Freundlich descreve o equilíbrio em superfícies heterogêneas, consequentemente não assume a capacidade de monocamada. ${ }^{35}$

O modelo pode ser representado pela expressão linear da Equação 7:

$\log \mathrm{q}_{\mathrm{e}}=\log \mathrm{K}_{\mathrm{f}}+1 / n \log C_{e}$

onde $\mathrm{C}_{\mathrm{e}}$ é a concentração do RL16 no tempo de equilíbrio $\left(\mathrm{mg} \mathrm{L}^{-1}\right)$, $\mathrm{q}_{\mathrm{e}}$ é a quantidade adsorvida do RL16 no tempo de equilíbrio $\left(\mathrm{mg} \mathrm{g}^{-1}\right)$ $\mathrm{e}, \mathrm{K}_{\mathrm{f}}$ e $\mathrm{n}$ são constantes relacionadas com a capacidade de adsorção e a intensidade de adsorção, respectivamente.

Os parâmetros das isotermas de Langmuir e Freundlich foram determinados pelos coeficientes lineares e angulares dos gráficos lineares (Tabela 2). Os dados experimentais ajustaram-se melhor ao modelo da isoterma de Langmuir evidenciado pelos valores do coeficiente de determinação $\left(\mathrm{R}^{2}\right)$ e pela comparação dos valores experimentais da adsorção do RL16 sobre a ZM-2 com os valores estimados pelos modelos de Langmuir e Freundlich (Figura 4). A capacidade de adsorção máxima do RL16 sobre ZM-2 foi de $1,14 \mathrm{mg} \mathrm{g}^{-1}$.

Tabela 2. Parâmetros das isotermas de Langmuir e Freundlich do RL16 pela ZM-2

\begin{tabular}{lccccc}
\hline Langmuir & & \multicolumn{3}{c}{ Freundlich } \\
\hline $\mathrm{Q}_{\mathrm{o}}(\mathrm{mg} / \mathrm{g})$ & $\mathrm{b}(\mathrm{L} / \mathrm{mg})$ & $\mathrm{R}^{2}$ & $\mathrm{~K}_{\mathrm{f}}{ }^{*}$ & $\mathrm{n}$ & $\mathrm{R}^{2}$ \\
1,14 & 2,03 & 0,992 & 0,62 & 2,55 & 0,823 \\
\hline
\end{tabular}

(*) $(\mathrm{mg} / \mathrm{g})(\mathrm{L} / \mathrm{mg})^{1 / \mathrm{n}}$

\section{CONCLUSÃO}

Neste estudo, a zeólita sintetizada a partir das cinzas leves de carvão mineral - utilizado em usinas termelétricas - foi avaliada como adsorvente do corante RL16 em solução aquosa. Os estudos cinéticos mostraram que a adsorção de RL16 concorda com o modelo de pseudossegunda ordem. Os modelos de isotermas de adsorção de Langmuir e de Freundlich foram usados para descrever o equilíbrio de adsorção do RL16 pela ZM-2. Os dados mostraram melhor adequação ao modelo de Langmuir. A zeólita sintetizada a partir de cinzas leves de carvão mineral revelou ter potencial para ser utilizada como um material alternativo na remoção de cor de efluentes aquosos. Se não forem descartadas adequadamente, as cinzas leves de carvão, matéria-prima do adsorvente, são uma fonte contínua de poluição do meio ambiente.

\section{MATERIAL SUPLEMENTAR}

O material suplementar descrito a seguir está disponível gratuitamente em http://quimicanova.sbq.org.br na forma de arquivo PDF, com acesso livre: Tabela 1S: Características gerais do corante RL16; Figura 1S: Estrutura química do corante RL16; Figura 2S: Difratogramas de raios X da CL-2 e da ZM-2; Figura 3S: Fotograma MEV da zeólita de cinzas de carvão ZM-2; Figura 4S: Espectros na região do infravermelho da CL-2 e da ZM-2.

\section{AGRADECIMENTOS}

Ao Conselho Nacional de Desenvolvimento Científico e Tecnológico - CNPq pelo apoio financeiro e à Carbonífera do Cambuí Ltda., Figueira - PR pelo fornecimento de amostras de cinzas de carvão.

\section{REFERÊNCIAS}

1. htpp:// www.aneel.gov.br, acessada em Janeiro 2009.

2. Levandowski, J.; Kalkreuth, W.; Int. J. Coal Geol. 2009, 77, 269.

3. Izidoro, J. C.; Dissertação de Mestrado, Instituto de Pesquisas Energéticas e Nucleares, Brasil, 2008.

4. Jha, V. K.; Matsuda, M.; Miyake, M.; J. Ceram. Soc. Jpn. 2008, 116, 167.

5. Fungaro, D. A.; Silva, M. G.; Quim. Nova 2002, 25, 1081.

6. Fungaro, D. A.; Flues, M. S-M; Celebroni, A. P.; Quim. Nova 2004, 27, 582.

7. Fungaro, D. A.; Izidoro, J. C. Tratamento de efluentes industriais usando material zeolítico preparado com cinzas de carvão. Em Água - Fonte da Vida / Prêmio Jovem Cientista e Prêmio Jovem Cientista do Futuro. Gráfica e Editora Comunicação Impressa: Porto Alegre, 2004, p. 129.

8. Fungaro, D. A.; Izidoro, J. C.; Almeida, R. S.; Eclética Quim. 2005, 30, 31.

9. Fungaro, D. A.; Izidoro, J. C.; Quim. Nova 2006, 29, 735.

10. Izidoro, J. C.; Fungaro, D. A.; Rev. Bras. Pesq. Des. 2007, 9, 101.

11. Karagozoglu, B.; Tasdemir, M.; Demirbas, E.; Kobya, M.; J. Hazard. Mater. 2007, 147, 297.

12. Ozdemir, O.; Armagan, B.; Turan, M.; Çelik, M. S.; Dyes Pigm. 2004, $62,49$.

13. Höller, H.; Wirsching, G. U.; Fortschr. Miner. 1985, 63, 21.

14. Querol, X.; Plana, F.; Alastuey, A.; Lopez-Soler, A.; Fuel 1997, 76, 793.

15. Querol, X.; Moreno, N.; Umaña, J. C.; Alastuey, A.; Hernández, E.; Lopez-Soler, A.; Plana, F.; Int. J. Coal Geol. 2002, 50, 413.

16. Rayalu, S. S.; Bansiwal, A. K.; Meshram, S. U.; Labhsetwar, N.; Devotta, S.; Catal. Sur. Asia 2006, 10, 74.

17. Guisnet, M.; Ribeiro, F. R.; Zeólitos - um nanomundo ao serviço da catálise, Fundação Calouste Gulbenkian: Lisboa, 2004.

18. Ojha, K.; Pradhan, N. C.; Samanta, A. N.; Bull. Mater. Sci. 2004, 27, 555 . 
19. Thinakaran, N.; Panneerselvam, P.; Baskaralingam, P.; Elango, D.; Sivanesan, S.; J. Hazard. Mater. 2008, 158, 142.

20. Uzun, I.; Dyes Pigm. 2006, 70, 76.

21. Guarantini, C. C. I.; Zanoni, M. V. B.; Quim. Nova, 2000, 23, 71.

22. Moreau, L.; Goossens, A.; Contact Dermatitis 2005, 53, 150.

23. Toor, A. P.; Verma, A.; Jotshi, C. K.; Bajpai, P. K.; Singh, V.; Dyes Pigm 2006, 68, 53 .

24. Schneider, K.; Hafner, C.; Jager, I.; J. Appl. Toxicol. 2004, 24, 83.

25. Santhy, K.; Selvapathy, P.; Biores. Technol. 2006, 97, 1329.

26. Biniwale, R.; Rayalu, S.; Hazan, M. Z.; J. Sci. Ind. Res. 2001, 60, 574.

27. Perrich, J. R.; Activated carbon adsorption for waste water treatment, CRC Press: Boca Raton, 1981.

28. Propocki, A.; Cardoso, A. M.; Ligabue, R. A.; Azevedo, A. M. N.; Villwock, J. A.; Pires, M.; XVIII Congresso Brasileiro de Engenharia e Ciência dos Materiais, Porto de Galinhas, Brasil, 2008.

29. Mall, I. D.; Srivastava, V. C.; Agarwal, N. K.; Dyes Pigm. 2006, 69, 210.

30. Vadivelan, V.; Kumar, K. V.; J. Colloid Interface Sci. 2005, 286, 90.

31. Lagergren, S.; Handlingar 1898, 24, 1.
32. Ho, Y. S.; McKay, G.; Can. J. Chem. Eng. 1998, 76, 822.

33. Ho, Y. S.; Wase, D. A. J.; Forster, C. F.; Environ. Technol. 1996, 17, 71.

34. Weber, W. J.; Morris, J. C.; J. Sanit. Eng. Div. 1963, 89, 31.

35. Dizge, N.; Aydiner, C.; Demirbas, E.; Kobya, M.; Kara, S.; J. Hazard. Mater. 2008, 150, 737.

36. Allen, S. J.; McKay, G.; Khader, K. Y. H.; Environ. Pollut. 1989, 56, 39.

37. Ho, Y. S.; McKay, G.; Chem. Eng. J. 1998, 70, 115.

38. Bhattacharyya, K. G.; Sharma, A.; Dyes Pigm. 2005, 65, 51.

39. Dabrowski, A.; Adv. Colloid Interf. Sci. 2001, 93, 135.

40. Giles, C. H.; MacEwan, T. H.; Nakhwa, S. N.; Smith, D.; J. Chem. Soc. 1960, 3973.

41. Giles, C. H.; Smith, D.; Huitson, A.; J. Colloid Interface Sci. 1974, 47, 755.

42. Khalaf, M.; Tese de Doutorado, Heinrich-Heine Universitat, Alemanha, 2003.

43. Eastoe, J.; Dalton, J. S.; Adv. Colloid Interf. Sci. 2000, 85, 103.

44. Chatterjee, S.; Chatterjee, S.; Chatterjee, B. P.; Guha, A. K.; Coll. Surf. A 2007, 299, 146. 


\section{ADSORÇÃO DO CORANTE REATIVO LARANJA 16 DE SOLUÇÕES AQUOSAS POR ZEÓLITA SINTÉTICA}

Terezinha Elizabeth Mendes de Carvalho, Denise Alves Fungaro* e Juliana de Carvalho Izidoro

Centro de Química e Meio Ambiente, Instituto de Pesquisas Energéticas e Nucleares, Av. Prof. Lineu Prestes, 2242, 05508-000 São Paulo - SP, Brasil<smiles>CC(=O)Nc1ccc2cc(S(=O)(=O)O)c(N=Nc3ccc(S(=O)(=O)CCOS(=O)(=O)O[Na])cc3)c(O)c2c1</smiles>

Figura 1S. Estrutura química do corante RL16

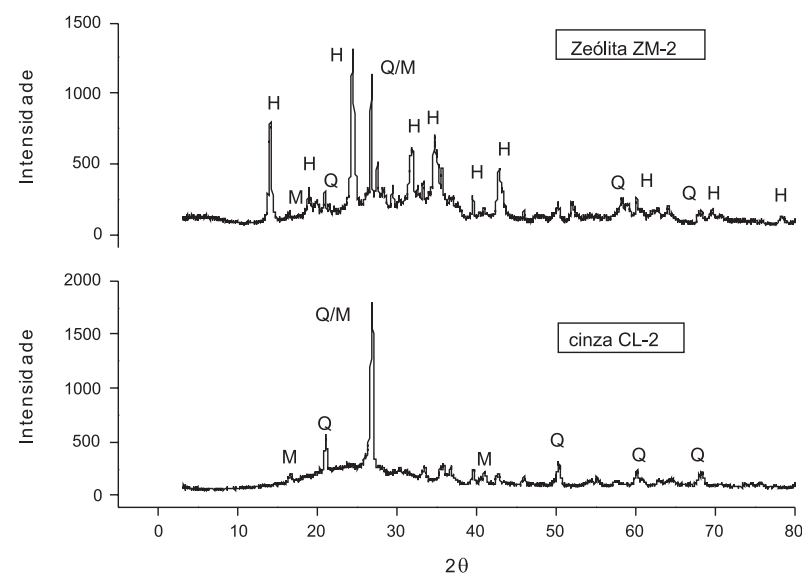

Figura 2S. Difratogramas de raios $X$ da $C L-2$ e da ZM-2: Q=quartzo; $M=$ mulita; $H=$ Hidroxissodalita

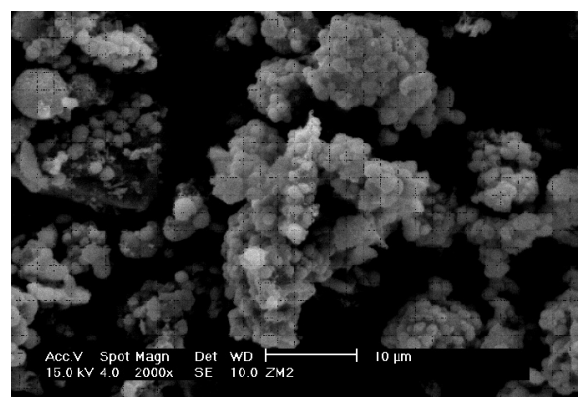

Figura 3S. Fotograma de MEV da zeólita de cinzas de carvão (ZM-2)
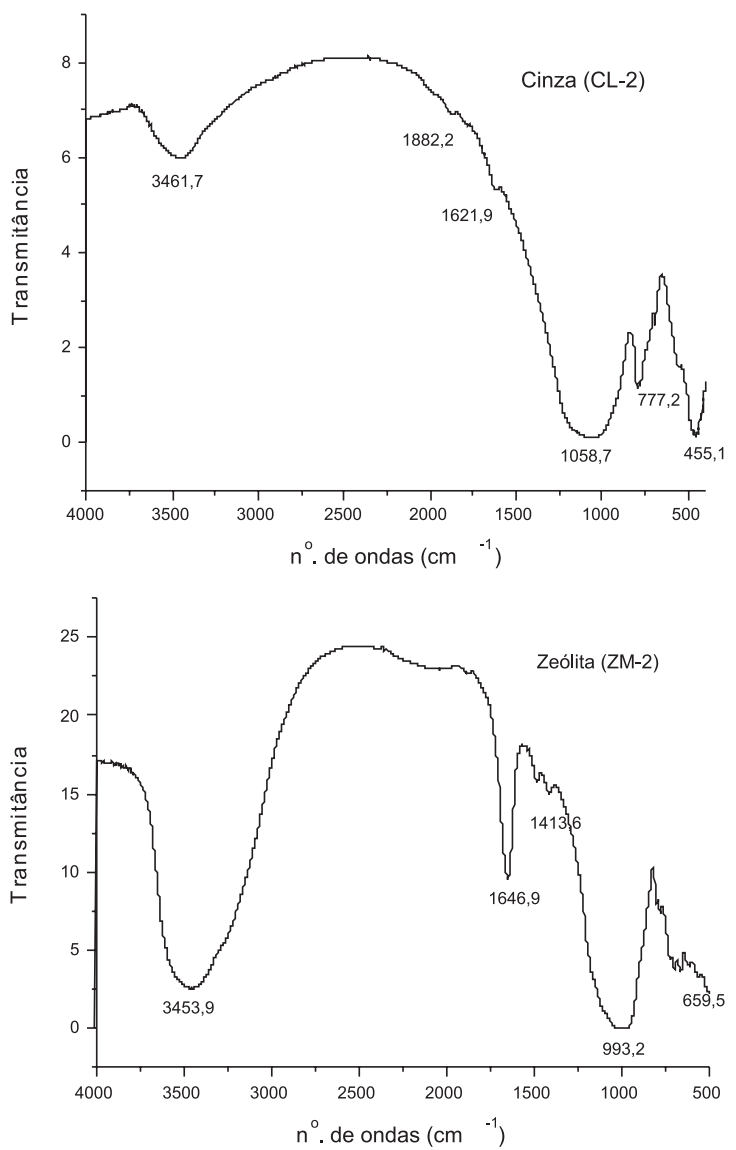

Figura 4S. Espectros na região do infravermelho da CL-2 e da ZM-2

*e-mail: dfungaro@ipen.br 